\title{
Transapical transcatheter aortic valve implantation in a high-risk patient with aortic and mitral regurgitation: usage of the JenaValve ${ }^{\mathrm{TM}}$ system
}

\author{
Suleyman Surer, Ilker Ince, Ibrahim Duvan, Cengizhan Bayyurt, Ugursay Kiziltepe \\ Department of Cardiovascular Surgery, Diskapi Yildirim Beyazit Training and Research Hospital, Ankara, Turkey
}

\begin{abstract}
JenaValve ${ }^{\mathrm{TM}}$ system is a second-generation transcatheter heart valve that provides a successful deployment not only in calcified stenotic aortic valves but also in non-calcified, severe regurgitant aortic valves. We performed a transapical transcatheter aortic valve implantation procedure using this system without any procedure-related complications in a 75-year-old woman with multiple co-morbidity factors, who had both mitral and aortic regurgitation, and low ejection fraction.
\end{abstract}

Eur Res J 2016;2(3):219-221

Keywords: Aortic regurgitation; mitral regurgitation; transcatheter aortic valve implantation; transapical; JenaValve ${ }^{\mathrm{TM}}$ system

\section{Introduction}

Indication criteria of transcatheter aortic valve implantation (TAVI) in patients with severe aortic stenosis and the contraindications or risk profile for open heart surgery have been determined but treatment of regurgitation of both mitral and aortic valves in a high risk patient with very low ejection fraction is a surgical challenge. Regurgitation in aortic valve without stenosis presents a subject of debate for TAVI and increases the risk of dislocation depending on the lack of the calcifications needed for perfect deployment of transcatheter heart valves. In addition to existing co morbidities, the combination of afterload mismatch and decreased ejection fraction following the relief of mitral regurgitation may increase the perioperative mortality and morbidity. An ejection fraction lower than $30 \%$ is accepted as an inoperability criterion in patients with mitral regurgitation by some authors. Severe aortic regurgitation related with left ventricle (LV) volume overload and resultant LV dilatation may contribute to the development of mitral regurgitation and relief of aortic insufficiency via a less invasive way may decrease the mitral regurgitation by decompression and reverse remodeling of the dilated LV. The JenaValve $^{\mathrm{TM}}$ is the only TAVI system approved for both aortic regurgitation and stenosis because the JenaValve ${ }^{\mathrm{TM}}$ prosthesis represents a clip fixation on the native aortic valve cusps providing perfect 
deployment for the transcatheter heart valves even in the lack of calcifications $[1,2]$.

\section{Case Presentation}

A 75 year-old woman with orthopnea was admitted to our institution with symptomatic severe aortic and moderate-severe mitral regurgitation (Figure 1). She had a functional class of NYHA IV. In addition to co-morbidities like diabetes, chronic renal failure, chronic obstructive pulmonary disease and history of stroke; she had an ejection fraction of $30 \%$, a dilated LV (LV end-diastolic diameter: $6.5 \mathrm{~cm}$ ) and an elevated systolic pulmonary artery pressure of 70 mmHg with a logistic EUROSCORE of $38 \%$. Those factors made us think an open surgical repair of both valves would be too risky and a palliative approach with the relief of aortic regurgitation alone could be a better option with a TAVI procedure.

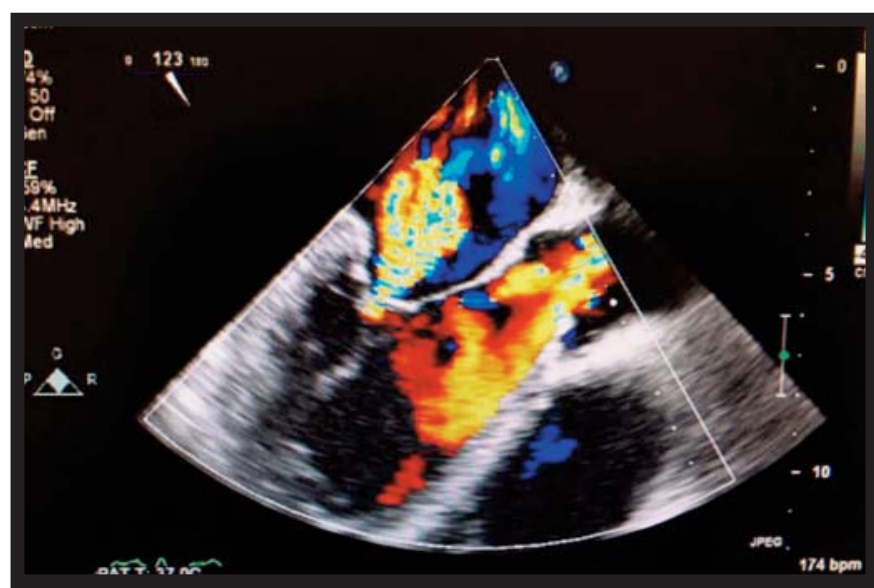

Figure 1. Preoperative echocardiographic view of the regurgitant aortic and mitral valves

The JenaValve ${ }^{\mathrm{TM}}$ implantation was performed under general anesthesia by a multidisciplinary approach team including cardiac surgeons, cardiologists and anesthesiologists. Transapical access was performed via a left anterolateral minithoracotomy and purse-string sutures were carried out to the left ventricular apex. A $27 \mathrm{~mm}$ JenaValve $^{\mathrm{TM}}$ (JenaValve Technology GmbH, Munich, Germany) was implanted and the patient tolerated the procedure well (Figures 2 and 3). No adverse events existed related with the procedure and postoperative period of the patient was uneventful.

Her immediate and postoperative $2^{\text {nd }}$ month echocardiography studies depicted a reduction in the level of mitral regurgitation, the size of LV (end- diastolic diameter from 6.5 to $5.8 \mathrm{~cm}$ ) and systolic pulmonary artery pressure (from $70 \mathrm{mmHg}$ to 45 $\mathrm{mmHg}$ ) in addition to improvement of her functional class from NYHA IV to II. Postoperative VARC 2 (Valve Academic Research Consortium) endpoints were evaluated and there were no symptoms or signs of the complications defined [3].

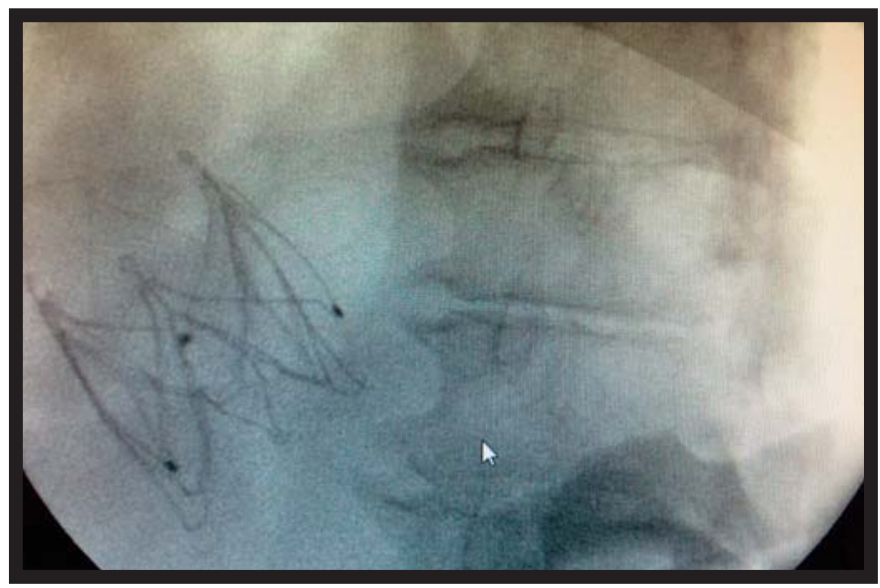

Figure 2. Intraoperative image of the completed transapical TAVI procedure by JenaValve ${ }^{\mathrm{TM}}$

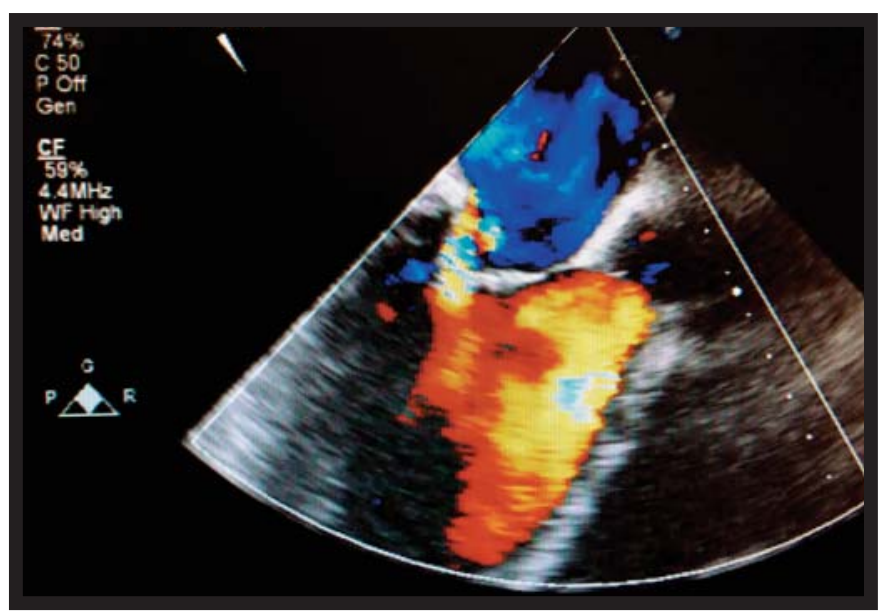

Figure 3. Postoperative echocardiographic view

\section{Discussion}

In respect of the guidelines on the management of valvular heart disease; aortic valve surgery is advised to be performed (Class IB) in severe aortic regurgitation for symptomatic patients and in the presence of ejection fraction $<50 \%$, LVEDD $>70 \mathrm{~mm}$, LVESD $>50 \mathrm{~mm}$ as the prognostic signs of impaired LV [1].

In our case, symptoms and signs of operable severe aortic regurgitation were present but unfortunately, she could not be an optimal candidate 
for an open surgical procedure because of having multiple major co-morbidity factors additionally to severe non-calcified aortic regurgitation concomitant with mitral regurgitation. Non calcified, severe aortic regurgitation was accepted as a relative contraindication for TAVI before, because both Medtronic Core Valve and Edwards Sapien transcatheter heart valves could offer only a limited success in the treatment of non-calcified aortic regurgitation with a serious risk of annular rupture, incomplete valve expansion, insufficient anchoring, valve embolization or residual paravalvular regurgitation because either of the transcatheter heart valves were produced to be implanted on a calcified aortic annulus safely via radial force of the prosthesis [2].

The JenaValve ${ }^{\mathrm{TM}}$ system is a second-generation transcatheter heart valves that provides a successful deployment not only in calcified, stenotic aortic valves but also in non-calcified, severely regurgitant aortic valves by its feeler guided positioning and secure clip fixation mechanism to the native aortic valve cusps. Calcium spots occurring on regurgitant aortic valve may complicate the procedure by causing an incomplete stent expansion because the JenaValve ${ }^{\mathrm{TM}}$ system carries less radial force than a balloonexpandable transcatheter heart valves [3].

In our patient, existence of severe aortic regurgitation was accompanying with moderatesevere mitral regurgitation. We consulted that severe aortic regurgitation might have contributed to severity of mitral regurgitation. As a result; due to the presence of multiple co-morbidities in this case, we considered that amelioration of aortic regurgitation alone and eventual decompression of distended LV might attenuate the level of mitral regurgitation and the patient could have tolerated a TAVI procedure by a second-generation transcatheter heart valve JenaValve ${ }^{\mathrm{TM}}$ system better than a surgical repair/replacement for both aortic and mitral valve pathologies.

We also chose to perform the TAVI procedure using JenaValve ${ }^{\mathrm{TM}}$ system for the advantages about the complications seen less than the other TAVI procedures, such as thromboembolic and cerebrovascular events, conduction disturbances, annular dilation or rupture by the exclusion of oversizing and valvuloplasty as well as cancelling rapid ventricular pacing during the deployment of the valve. The procedure can be performed while heart is beating and this makes the procedure safer than the others especially in patients with low cardiac output as our patient [3].

\section{Conclusion}

In high risk setting of combined aortic and mitral valve regurgitations and low ejection fraction, a suboptimal treatment option with a transapical TAVI procedure by a second-generation transcatheter heart valves, the JenaValve ${ }^{\mathrm{TM}}$ system alone may be a more reasonable and safer option than open surgical repair/replacement of both regurgitant valves.

\section{Informed consent}

Written informed consent was obtained from the patient for the publication of this case report.

\section{Conflict of interest}

The authors declared that there are no potential conflicts of interest with respect to the research, authorship, and/or publication of this article.

\section{References}

[1] Joint Task Force on the Management of Valvular Heart Disease of the European Society of Cardiology (ESC); European Association for Cardio-Thoracic Surgery (EACTS), Vahanian A, Alfieri O, Andreotti F, Antunes MJ, Baron-Esquivias G, Baumgartner $\mathrm{H}$, et al. Guidelines on the management of valvular heart disease (version 2012). Eur Heart J 2012;33:2451-96.

[2] Holmes DR Jr, Mack MJ, Kaul S, Agnihotri A, Alexander KP, Bailey SR, et al. 2012 ACCF/ AATS/SCAI/STS expert consensus document on transcatheter aortic valve replacement. J Am Coll Cardiol 2012;59:1200-54.

[3] Seiffert M, Bader R, Kappert U, Rastan A, Krapf S, Bleiziffer $\mathrm{S}$, et al. Initial German experience with transapical implantation of a second-generation transcatheter heart valve for the treatment of aortic regurgitation. JACC Cardiovasc Interv 2014;7:1168-74. 